\title{
Análisis con elemento finito de los esfuerzos expansivos por corrosión en las estructuras de concreto reforzado
}

\author{
Finite Element Model for Expansive Stress due \\ to Corrosion of Reinforced Concrete Structures
}

Castorena-González J.H.

Facultad de Ingeniería Mochis

Universidad Autónoma de Sinaloa. Los Mochis, Sin., México

E-mail:kstor28@yahoo.com.mx

Almeraya-Calderón F.

Departamento de Física

de Materiales/Grupo corrosión

Centro de Investigación en Materiales Avanzados, S.C.

Chihuahua, Chih., México

E-mail: facundo.almeraya@cimav.edu.mx

Almaral-Sánchez J.L.

Facultad de Ingeniería Mochis

Universidad Autónoma de Sinaloa.

Los Mochis, Sin., México

E-mail:jalmaral@gmail.com
Calderón-Guillén J.A.

Facultad de Ingeniería Mochis

Universidad Autónoma de Sinaloa.

Los Mochis, Sin., México

E-mail:cajoel_99@yahoo.com

Gaona-Tiburcio C.

Departamento de Física

de Materiales/Grupo corrosión

Centro de Investigación en Materiales Avanzados, S.C.

Chihuahua, Chih., México

E-mail: citlalli.gaona@cimav.edu.mx

Martínez-Villafañe A.

Departamento de Física

de Materiales/Grupo corrosión

Centro de Investigación en Materiales Avanzados, S.C.

Chihuahua, Chih., México

E-mail:martinez.villafane@cimav.edu.mx

Información del artículo: recibido: noviembre de 2006, aceptado: agosto de 2010

\section{Resumen}

La corrosión en el acero de refuerzo es un problema que disminuye la vida útil en las estructuras de concreto reforzado, además de comprometer su seguridad estructural. En los modelos disponibles para estimar el efecto mecánico de la corrosión, se supone que el acero corroído, a través de los óxidos que crecen a su alrededor, ejercen una presión sobre el concreto circundante suponiendo un problema de esfuerzos o deformaciones planas. En el presente trabajo, se modela el problema con elemento finito tridimensional a partir de un experimento sobre un cilindro de concreto reforzado sometido a corrosión acelerada, instrumentado para medir indirectamente la presión en la interfase acero-concreto. De los resultados obtenidos se concluye que el efecto de la longitud de acero corroído, longitud anódica, tiene un efecto significativo sobre la magnitud de la presión en la interfase acero-concreto, hecho que puede ser utilizado para mejorar los modelos existentes.

\section{Descriptores}

- corrosión

- acero de refuerzo

- estructuras de concreto reforzado

- longitud anódica

- elemento finito

- interfase acero-concreto 


\begin{abstract}
The corrosion in the reinforcement steel is a problem that diminishes the useful life of reinforced concrete structures, besides committing its structural security. In the available models to estimate the mechanical effect of the corrosion, it is assumed that the corroded steel, through the oxides that grow to its surroundings, exercises a pressure on the surrounding concrete supposing a problem of plane stress or plane strain. In this work, the problem is modeled with three-dimensional finite element starting from an experiment on a subjected cylinder to accelerated corrosion, with strain gages to measure the pressure indirectly in the interface steel-concrete. From the results obtained it can be concluded that the effect of the length of corroded steel, anodic length, has a significant effect on the magnitude of the pressure in the interface steel-concrete, fact that can be used to improve the existing models.
\end{abstract}

Keywords

- corrosion

- reinforcement

- reinforced concrete structures

- anodic length

- finite element

- interface steel-concrete

\section{Introducción}

La corrosión del acero en las estructuras de concreto reforzado (CR), es un fenómeno que es considerado como una de las causas principales de deterioro estructural (Cabrera, 1996 y Raupach et al., 2001). La diferencia entre la corrosión del acero expuesto al medio ambiente y la del acero incluido en el concreto reforzado, es que en este último, el concreto sirve como una barrera física y química que protege al acero (Jarrah et al., 1995); por un lado, existe una porción de concreto denominada recubrimiento, que impide el paso de agentes dañinos para el acero. Como barrera química, durante el proceso de hidratación del cemento, se genera un ambiente altamente alcalino ( $\mathrm{pH}$ entre 13 y 13.8), principalmente debido a la producción de $\mathrm{Ca}(\mathrm{OH})_{2}$, así como $\mathrm{NaOH}$ y $\mathrm{KOH}$, los cuales están presentes en la pasta de cemento. En este ambiente alcalino los componentes termodinámicamente estables del acero, $\mathrm{Fe}_{3} \mathrm{O}_{4} \mathrm{O} \gamma-\mathrm{Fe}_{2} \mathrm{O}_{3}$ (Batis et al., 1999), forman una delgada capa de óxido protectora, denominada capa pasiva, que se forma de manera espontánea con espesor de unos cuantos nanómetros, por lo que es muy difícil estudiar sus propiedades. Sin embargo, la barrera física de concreto no es impermeable y pueden penetrar a través de sus poros agentes nocivos que llegan a destruir esa capa pasiva (Cabrera et al., 1995).

Por lo general, una vez que los iones Fe se liberan del acero, reaccionan con el oxígeno circundante y forman óxidos más estables químicamente, los cuales se van depositando en la superficie del acero como se muestra en al figura 1. Ahí se tiene un fenómeno similar a una celda electrolítica, donde la parte sana actúa como cátodo y la parte corroída como ánodo. Cuando los óxidos siguen creciendo, al tener menor densidad volumétrica que el acero original (con volumen de hasta 1.7 y 6.25 veces al del acero), ya no es posible que ocupen el mismo lugar que les correspondía en el acero, por lo que se expanden alrededor del refuerzo originando esfuerzos, los cuales al ser más grandes que los de tensión del concreto, provocan que éste se agriete y en casos severos hasta sufrir un desprendimiento del mismo (Pantazopoulou et al., 2001), causando que las condiciones de servicio, así como la capacidad portante de la estructura se vean reducidas (Lee et al., 2002 y Chang, 2003). Dependiendo de la localización del ánodo y el cátodo, la corrosión del refuerzo puede ocurrir ya sea en una microcelda, donde las reacciones anódica y catódica prácticamente se dan en el mismo lugar, o bien en una macrocelda, en donde se distinguen perfectamente al área corroída del refuerzo, ánodo, y a la parte no corroída, cátodo, (González et al., 1994 y Elsener, 2002).

La figura 2 muestra estos casos. El caso de la macrocelda es de gran importancia, ya que la reducción de la sección del refuerzo puede acelerarse debido a la relación tan grande entre áreas catódica y anódica, produciendo tasas de corrosión muy altas (Raupach et al., 2001). En la actualidad, gran parte de los trabajos de investigación han tratado sobre corrosión aproximadamente uniforme (microcelda) y los pocos casos de estudio en que se considera una fracción del acero corroída (macrocelda) se han realizado de manera experimental (Torres-Acosta et al, 2004). Puesto que en todo programa de mantenimiento y reparación de estructuras de concreto reforzado la identificación y medida del agrietamiento por corrosión es de suma importancia, se desarrolla el presente trabajo a fin de proporcionar información adicional sobre el efecto de la presión en la interfase acero corroído y concreto mediante un modelo de elemento finito tridimensional. 


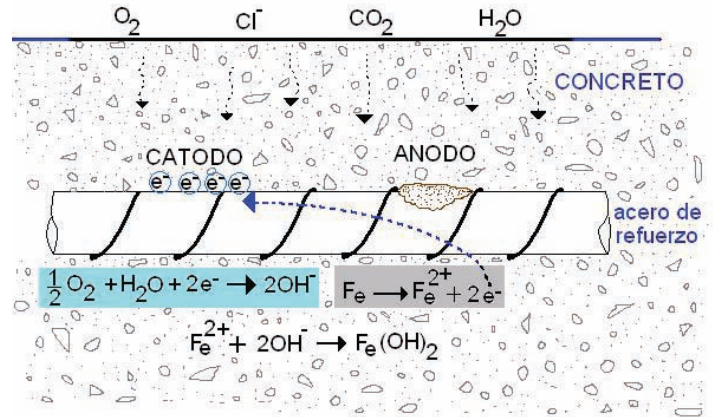

Figura 1. El proceso de corrosión en el acero de refuerzo. Como proceso electroquímico, existen reacciones anódica $\mathrm{Fe} \rightarrow \mathrm{Fe}^{2+}+2 \mathrm{e}^{-}$y catódica $1 / 2 \mathrm{O}_{2}+\mathrm{H}_{2} \mathrm{O}+2 \mathrm{e}^{-} \rightarrow 2 \mathrm{OH}^{-}$y un flujo de electrones del ánodo al cátodo.

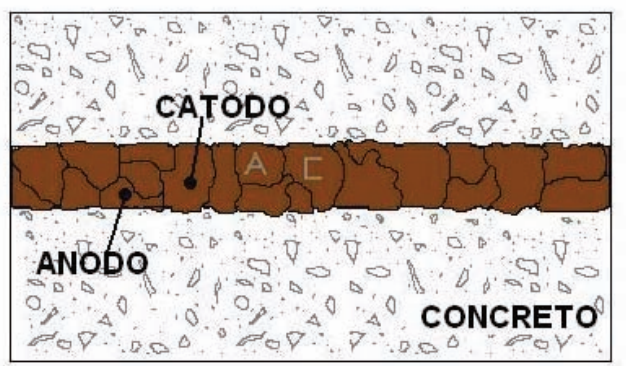

a)

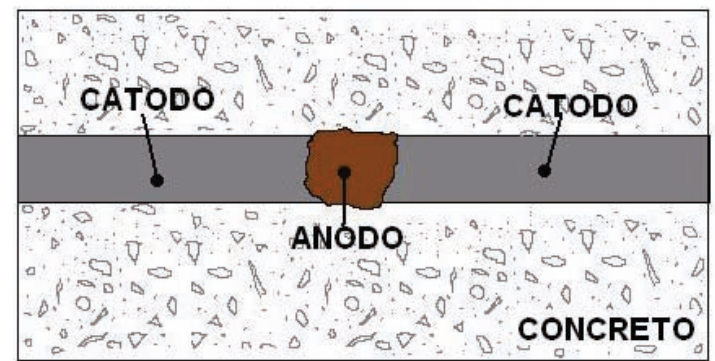

b)

Figura 2. Según la relación entre las áreas corroídas (ánodo) y sanas (cátodo) del refuerzo, el proceso de corrosión puede darse como una microcelda a), o macrocelda b).

\section{Modelación con elemento finito}

El agrietamiento del concreto se debe a la expansión de los productos de corrosión y para su predicción se han desarrollado algunos modelos (Bhargava et al., 2005); sin embargo, los resultados obtenidos no son satisfactorios. Algunas de las limitantes pudieran ser las condiciones de frontera alrededor del orificio ocupado por el acero corroído, ya que se considera al concreto que rodea al refuerzo como si fuera un cilindro de pared grue- sa sometido a presión interna, debida a los óxidos. Además, se desprecia la resistencia residual a tensión del concreto agrietado en el recubrimiento. En esta sección, se propone representar el acero corroído como un elemento estructural que es forzado a ocupar el volumen dentro de un cilindro hueco de concreto, cuyo diámetro interior es menor que el diámetro de la barra corroída y que una vez que las dos partes se han acoplado, se puede obtener la presión ejercida entre ellas. El desplazamiento relativo entre los diámetros, $\delta$, representa la capa de óxido necesaria para producir esfuerzos tangenciales iguales a la resistencia máxima a tensión del concreto e iniciar el agrietamiento en la interfase acero-concreto. Resolviendo entonces el problema mediante Teoría de Elasticidad (Timoshenko et al., 1970) se obtiene el valor de $\delta$ el cual está dado por la ecuación 1.

$\delta=2 r_{0} P_{s}\left[\frac{1-v_{a}}{E_{a}}+\frac{\left(1+v_{c}\right) R_{c}^{2}+\left(1-v_{c}\right) r_{0}^{2}}{E_{h}\left(R_{c}^{2}-r_{0}^{2}\right)}\right]$

$E_{a} \mathrm{y} v_{a}$ son los módulos de elasticidad y Poisson del acero de refuerzo, $E_{c}$ y $v_{c}$ los correspondientes al concreto, $r_{0}$ es el radio inicial del acero, $R_{c}$ es la distancia desde el centro del refuerzo a la superficie libre más cercana del recubrimiento. $P_{g}$ es la presión radial en la interfase acero-concreto y se obtiene mediante la ecuación 2, donde $f_{t}$ es la resistencia a tensión del concreto.

$f_{1}=\left[\frac{R_{c}^{2}+r_{0}^{2}}{R_{c}^{2}-r_{0}^{2}}+v_{c}\right] P_{s}$

En los cálculos de $\delta$ y $P_{g}$ con las ecuaciones anteriores, se supone que el concreto no está agrietado. Una vez que los óxidos continúan creciendo, esto es, a mayores valores de $\delta$, entonces el concreto se agrieta; sin embargo, aún agrietado se supone que posee alguna resistencia adicional a tensión. Este proceso será modelado con elemento finito mediante el paquete ANSYS, donde el proceso de agrietamiento será bajo el concepto de smeared crack, esto es, en lugar de suponer grietas discretas, se propone modificar las propiedades mecánicas del concreto de acuerdo a relaciones esfuerzo-deformación dadas para éste. La figura 3 muestra el concepto de smeared crack y las relaciones esfuerzo-deformación para el concreto, tanto a tensión como a compresión. La nomenclatura de la figura 3c corresponde a la de ANSYS, donde $T_{c}$ es un factor que toma en cuenta la relajación del esfuerzo. Para el trabajo desarrollado aquí, se da un valor de 0.9 . Tanto el valor de $T_{c}$ como la curva de la fi- 
gura 3d, fueron adoptados de un trabajo previo (Castorena et al., 2005) ya que con estos valores se resuelve el problema de convergencia. SOLID65 es el único elemento finito en ANSYS capaz de simular agrietamiento a tensión y aplastamiento a compresión en el concreto, consta de ocho nudos y tres grados de libertad en cada uno: desplazamientos lineales en direcciones $x, y \mathrm{y} z$. Para el caso de modelar con elemento finito la capa de óxido en la interfase acero-concreto, se utiliza el elemento de contacto CONTA173. Este elemento consta de cuatro nudos y se utiliza para representar el contacto y deslizamiento entre dos superficies rígidas y una deformable; las superficies rígidas corresponden al acero y concreto, la superficie deformable es la que se define por el elemento CONTA173. El acero de refuerzo es modelado como SOLID45, que al igual que SOLID65 es un elemento tridimensional con ocho nudos y tres grados de libertad, pero sin tomar en cuenta agrietamiento.

El análisis con elemento finito es un análisis numérico aproximado cuyos resultados, en cierta medida, dependen del tamaño de la malla de elementos finitos utilizada, por ello es que en cada aplicación se tiene que realizar un modelo calibrado, esto es, un modelo con el número determinado de elementos en donde, por más que se incrementen ya no cambia algún valor de interés (esfuerzo, desplazamiento, deformación, etcétera) en cierto punto. En este trabajo, para calibrar los modelos de elemento finito, se utiliza la solución teórica dada por la ecuación 1.

En la figura 4 se dan los resultados obtenidos para una sección transversal cilíndrica y otra rectangular, a la que se denomina viga, a efecto de mostrar que la ecuación 1 también puede ser utilizada para secciones rectangulares. Los datos para la obtención de los valores de las figuras 5 y 6 son: radio del refuerzo, $r_{0}=10.0 \mathrm{~mm}$, recubrimiento libre $\mathrm{C}=60 \mathrm{~mm}$, módulo de elasticidad del concreto $E_{h}=32390 \mathrm{MPa}$, módulo de elasticidad para el acero $E_{a}=205000 \mathrm{MPa}$, módulo de Poisson del acero $v_{a}=0.3 \mathrm{y}$ del concreto $v_{h}=0.24$. Los datos anteriores son ficticios y la importancia de los resultados mostrados en la figura 4 radica, para el presente trabajo, en que se utilizarán cilindros en la modelación con elemento finito para el cálculo de la presión en la interfase acero-concreto, por la simplicidad y además por las consideraciones de simetría.

\section{Desarrollo experimental}

Para esta prueba experimental se fabrican tres cilindros: un cilindro de concreto reforzado y dos de concreto simple. El cilindro reforzado tiene dimensiones de $150 \mathrm{~mm}$ de diámetro por $110 \mathrm{~mm}$ de altura y consta de una varilla de acero corrugado del No.8 $(25 \mathrm{~mm}$ de diámetro), de $30 \mathrm{~mm}$ de longitud. Dicho refuerzo fue maquinado, de manera que adquiere forma tubular, de radio interior de $8 \mathrm{~mm}$ y aprox. $4 \mathrm{~mm}$ de espesor. En el interior de dicho acero y a la mitad de su longitud se colocó un extensómetro, a efecto de monitorear las deformaciones durante el proceso de corrosión acelerada a la que sería sometido el cilindro. Los dos cilindros de concreto simple, de $150 \mathrm{~mm}$ de diámetro y $300 \mathrm{~mm}$ de altura, se ensayaron para obtener la resistencia del concreto utilizado en la prueba, siendo ésta en promedio a)
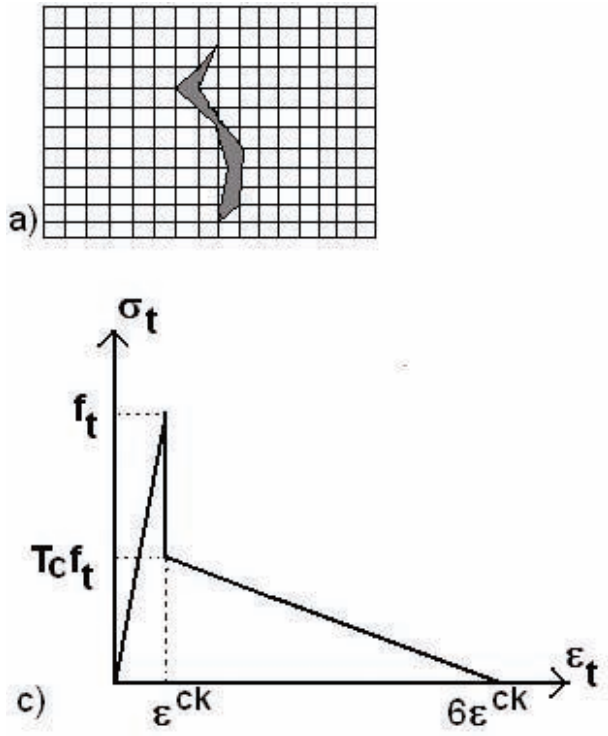

b)
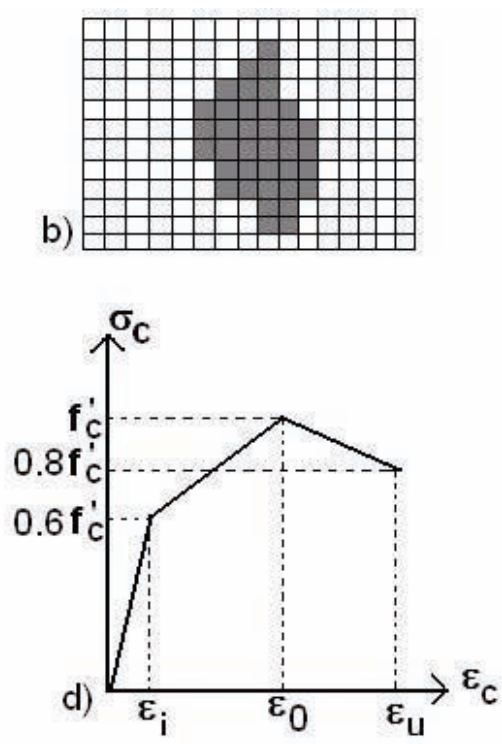

Figura 3. a) grieta discreta, b) smeared crack, c) y d) son curvas esfuerzo-deformación idealizadas para el concreto, a tensión y compresión, respectivamente. 


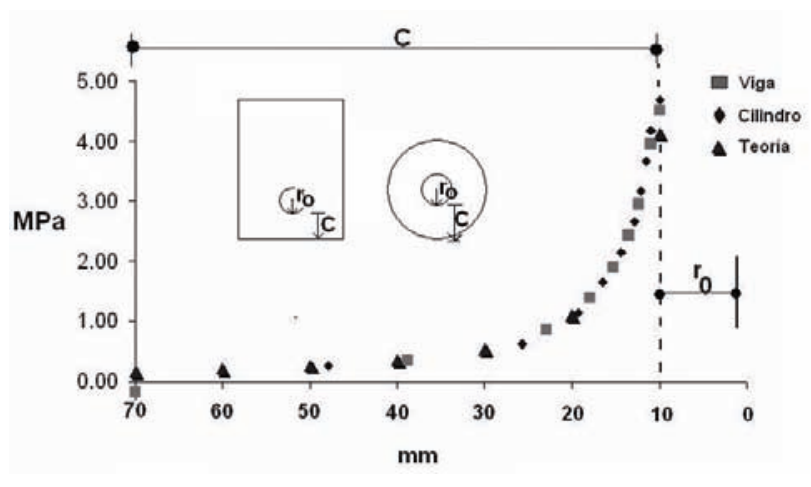

Figura 4. Aplicabilidad del modelo de elemento finito. La curva indica los esfuerzos tangenciales a lo largo del recubrimiento libre, $\mathrm{C}$.

de $245 \mathrm{~kg} . / \mathrm{cm}^{2}$ (24 MPa). La figura 5 muestra las dimensiones del cilindro y el modelo de elemento finito. La figura 6 ilustra el acero de refuerzo maquinado y el tipo de extensómetro utilizado.

\section{Resultados y discusión}

En la figura 7 se muestran los resultados de las deformaciones medidas en el interior del cilindro de acero
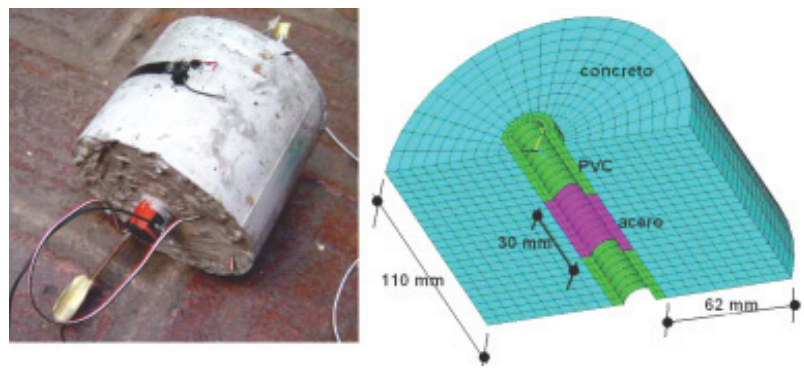

Figura 5. Geometría y modelo de elemento finito para el cilindro de pruebas.

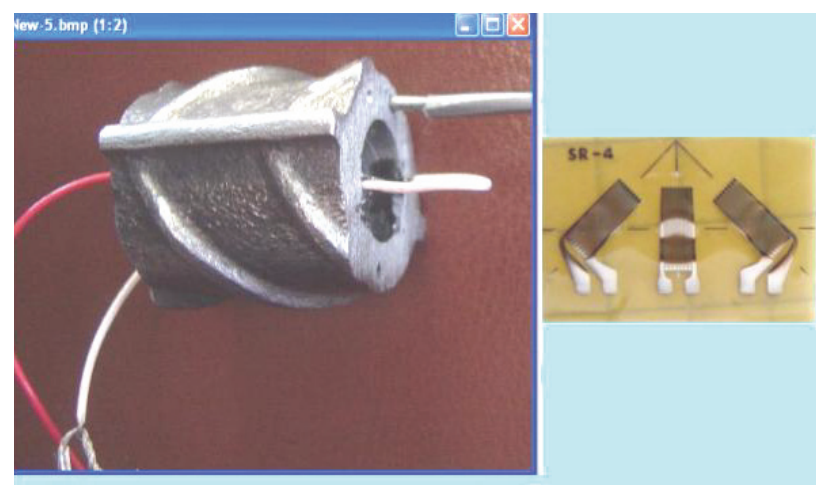

Figura 6. Acero de refuerzo instrumentado con extensómetro, a efecto de medir las deformaciones interiores producidas durante el proceso de corrosión acelerada. durante el período de corrosión. Para convertir las deformaciones medidas a presión se utilizan las ecuaciones dadas en la referencia (Torres-Acosta and Sagues, 2004). Las mediciones se realizaron a través de un medidor tipo 8692, de diez canales de lectura, distribuido por H. Tinsley \& Co. En dicha figura, se muestra un ligero quiebre inicial de la curva experimental, denotado por la línea con rombos, y es la presión que inicia el agrietamiento en la interfase acero-concreto. La línea con cuadros marca la cantidad de óxido sobre la curva en el que aparece la primera grieta visible por corrosión en la superficie del recubrimiento, en donde la presión en la interfase llega a alcanzar valores cercanos a la resistencia a compresión del concreto utilizado. Sobre la misma figura se incluyen los resultados con elemento finito. Para la modelación se requiere ingresar como dato la rigidez de contacto entre el acero corroído y el concreto, a través de las propiedades de los elementos de contacto CONTA173. Para el caso estudiado aquí, se obtuvo un valor de rigidez, denotado por FKN, de 0.26 veces a la del acero sano, esto es, de 546,000 kg/ $\mathrm{cm}^{2}$. Una vez que se obtienen resultados teóricos similares a los experimentales para una relación $\mathrm{C} / \mathrm{L}=2.2$, que correspondería a corrosión localizada, se realiza el ensayo con elemento finito para una relación teórica de $\mathrm{C} / \mathrm{L}=1.0$, que sería el caso de corrosión generalizada. En la gráfica se observa una disminución de la presión necesaria para que aparezca la primera grieta por corrosión en la superficie del recubrimiento libre. Este hecho se puede mostrar a través de experimentación y un análisis tridimensional.

\section{Conclusiones}

1. Es factible utilizar el método de elemento finito tridimensional para modelar el efecto mecánico de la

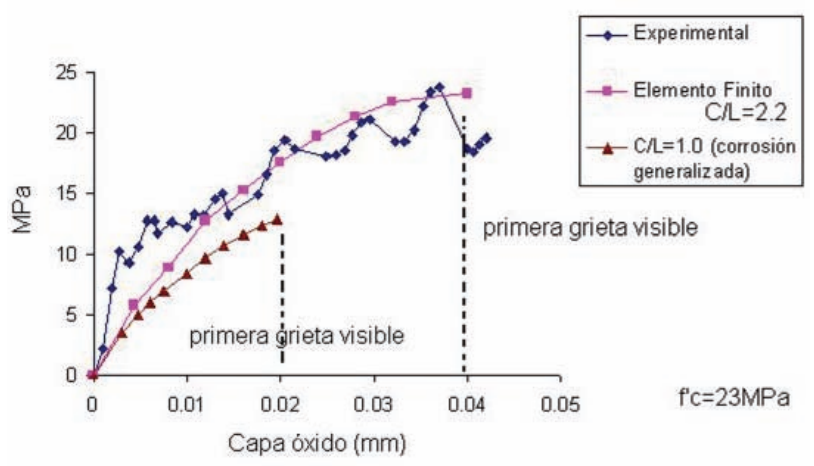

Figura 7. Relación entre presión-capa de oxido, en la interfase acero-concreto. 
corrosión del acero de refuerzo en las estructuras de concreto reforzado.

2. El efecto de los esfuerzos de expansión producido por el crecimiento de los óxidos alrededor del acero de refuerzo, se puede representar suponiendo al acero corroído como un elemento estructural que es forzado a ocupar un volumen mayor que el que ocupa el acero original, cuando se encuentra embebido en el concreto. De esta forma, cobra importancia la rigidez de contacto entre el acero corroído y el concreto.

3. Si se utiliza el software comercial de elemento finito ANSYS, el efecto de la presión de los productos de corrosión sobre el concreto puede representarse mediante los elementos de contacto CONTA173, con un valor de la rigidez de contacto FKN de 0.26 veces a la del acero original.

4. El valor de la presión de contacto entre el acero corroído y el concreto es afectado en gran medida por la magnitud de la longitud corroída, llegando a valores de la resistencia a compresión del concreto utilizado, para casos de corrosión localizada.

\section{Referencias}

Bhargava K, Ghosh A. Mori Y., Ramanujam S. Modeling of Time to Corrosion-Induced Cover Cracking in Reinforced Concrete Structures. Cement and Concrete Research, 35:2213-2218. 2005.

Batis G., Routoulas Th. Steel Rebars Corrosion Investigation with Strain Gages. Cement \& Concrete Composites, 21:163-171. 1999.

Cabrera G., Claisee A., Hunt, D. A Statistical Analysis of the Factors which Contribute to the Corrosion of Steel in Portland Cement and Silica Fume Concrete. Construction and Building Materials, 9:105-113. 1995.

Cabrera, G. Deterioration of Concrete due to Reinforcement Steel Corrosion. Cement \& Concrete Composites, 18:47-59. 1996.
Castorena J., Almeraya C., Velázquez I., Gaona C., Villafañe A. Modelación con elemento finito del agrietamiento por corrosión en vigas de concreto reforzado utilizando elementos de contacto mediante ANSYS. Conpat 2005, en: VIII Congreso Latinoamericano de Patología de la Construcción y X Congreso de Control de Calidad en la Construcción, Asunción (2005, Paraguay, 19-21 septiembre). Vol. I, pp. VI.43-VI.50

Chang J. Bond Degradation due to the Desalination Process. Construction and Building Materials, 17:281-287. 2003.

Elsener B. Macrocell Corrosion of Steel in Concrete -Implications for Corrosion Monitoring. Cement and Concrete Composites, 9:24:65-72. 2002.

González A., Benito M., Feliu S., Rodríguez P., Andrade, C. Suitability of Assessment Methods for Identifying Active and Passive Zones in Reinforced Concrete. Corrosion Engineering, 51:145-152. 1994.

Jarrah N., Al-Amoudi O., Maslehuddin M., Ashiru O., Al-Mana A. Electrochemical Behaviour of Steel in Plain and Blended Cement Concretes in Sulphate and/or Chloride Environments. Construction and Building Materials, 9:97-103. 1995.

Lee H., Noguchi T., Tomosawa F. Evaluation of the Bond Properties between Concrete and Reinforcement as a Function of the Degree of Reinforcement Corrosion. Cement and Concrete Research, 32:1313-1318. 2002.

Pantazopoulou J., Papulia D. Modeling Cover-Cracking due to Reinforcement Corrosion in RC Structures. Journal of Engineering Mechanics, 127:342-350. 2001.

Raupach M., Schiel P. Macrocell Sensor Systems for Monitoring of the corrosion Risk of the Reinforcement in Concrete Structures. NDTEE International, 34:435-442 2001.

Timoshenko S., Goodier J. Two-Dimensional Problems in Rectangular Coordinates. En:. International Student Edition. Theory of Elasticity. McGraw-Hill International Book Company. New York, USA, 1970, pp. 41-46.

Torres-Acosta A., Sagues A. Concrete Cracking by Localized Steel Corrosion-Geometric Effects. ACI Materials Journal, 101:501507. 2004. 


\section{Semblanza de los autores}

José Humberto Castorena-González. Es PITC titular "C" de la Universidad Autónoma de Sinaloa (UAS), Facultad de Ingeniería Mochis. Hizo sus estudios de Ingeniero Civil en el periodo 1983-1988. Obtuvo el grado de maestro en ingeniería estructural en el año 2001, en la Facultad de Ingeniería Civil, de la UAS. Obtuvo el grado de doctor en ciencia de materiales en el Centro de Investigación de Materiales Avanzados (CIMAV) en 2007 en el área de corrosión de estructuras de concreto reforzado. Otras áreas de interés son el análisis experimental de esfuerzos y elemento finito.

Facundo Almeraya-Calderón. Es investigador del Centro de Investigación de Materiales Avanzados (CIMAV). Nivel I del SNI. Ha realizado investigación en monitoreo de importantes obras, desde 1991, en las áreas de electroquímica y corrosión, y es consultor en corrosión para CONACYT, en México. Es miembro de NACE desde 1993, donde fungió como vicepresidente de 2001 a 2003, y actualmente es presidente del ALCONPAT sección México en el período de 2006 a 2008. Realizó sus estudios de ingeniería metalúrgica, UAM, maestría en metalurgia (corrosión) por la Universidad Nacional Autónoma de México (UNAM). Llevó a cabo sus estudios de doctorado en ciencia de los materiales en el CIMAV.

Jorge Luis Almaral-Sánchez. Es ingeniero civil egresado de la Universidad Autónoma de Sinaloa (UAS), 1982. Es PTIC-base-titular C de la UAS, Facultad de Ingeniería Mochis (ingresó desde 1984). En 2006 obtuvo distinciones de Perfil PROMEP, líder del cuerpo académico "Geotecnia, vías terrestres y materiales", revisor de la revista indexada JNCS y miembro del SNI a partir de 2007. Ha realizado investigación en calidad del concreto hidráulico, asfaltos modificados y en materiales híbridos orgánicos-inorgánicos. Hizo el curso "Diseño-proyecto-construcción de puentes", DEC-FI-UNAM, 1982-1983. Obtuvo el grado de maestro en ingeniería (vías terrestres), 1999 por la Universidad Autónoma de Chihuahua y de doctor en ciencias (materiales), 2005 por el CINVESTAV-Querétaro.

Joel Andrés Calderón-Guillén. Es PITC Titular "C" de la Universidad Autónoma de Sinaloa (UAS), Facultad de Ingeniería Mochis. Obtuvo el grado de doctor en ciencias (materiales) en 2006 por el CINVESTAV-Querétaro. Se desempeña en las áreas de modelación, propiedades y modificación de materiales.

Citlalli Gaona-Tiburcio. Es Investigadora del Centro de Investigación de Materiales Avanzados (CIMAV). Nivel 1 del SNI. Ha trabajado en las áreas de agrietamiento de corrosión por tensión y de corrosión influenciada por microbiología, que monitorea desde 1991 en México. Ella también trabaja como consultora de corrosión para CONACYT en México. Es miembro de NACE desde 1999, donde fungió como secretaria en la sección México de 2004 a 2005. Tiene maestría en ciencias (metalurgia) por la UNAM y doctorado en ciencia de los materiales egresada del CIMAV.

A. Martínez-Villafañe. Es investigador y encabeza el Departamento de Física de Materiales en el Centro de Investigación de Materiales Avanzados. Nivel III del SNI. Trabaja en las áreas de corrosión de alta-temperatura, recubrimientos metálicos, y monitoreo de corrosión. Es consultor de corrosión para CONACYT. Es miembro de NACE desde 1982, donde fue presidente de la sección México en 1993 y de 2001 a 2003. Tiene 26 años de experiencia en corrosión y control de corrosión. Tiene maestría en ciencia de los materiales por el Instituto de Politécnico Nacional en México y doctorado en ciencia de corrosión e ingeniería por el UMIST en el Reino Unido. 